\title{
Economic impact of pharmacist interventions on correction of stress-related mucosal damage prophylaxis practice
}

This article was published in the following Dove Medical Press journal:

ClinicoEconomics and Outcomes Research

\author{
Laleh Mahmoudi' \\ Rahim Mohammadi' \\ Ramin Niknam² \\ 'Department of Clinical Pharmacy, \\ School of Pharmacy, Shiraz University \\ of Medical Sciences, Shiraz, Iran; \\ ${ }^{2}$ Gastroenterohepatology Research \\ Center, Shiraz University of Medical \\ Sciences, Shiraz, Iran
}

\begin{abstract}
Introduction: Stress-related mucosal damage (SRMD) is described as the damage of gastric mucosa due to physiological stress that is a very common complication in critically ill patients. SRMD prophylactic medications are widely prescribed all over the world, while numerous studies have revealed that a large percentage of patients admitted to non-intensive care unit (ICU) services do not need to receive these medications. The aim of this study was to determine the frequency and type of medication errors and the economic impact of clinical pharmacist intervention on stress ulcer prophylaxis (SUP).

Methods: This prospective interventional study was conducted on adult patients admitted to internal, surgical, and critical care units at two large academic medical centers over 6 months Risk factors of stress ulcer were recorded daily during hospital stay, and appropriateness of SUP administration was assessed according to the American Society of Health-System Pharmacists (ASHP) criteria. An intervention was performed by a clinical pharmacist in the case of contradictions. The rate of inappropriate SUP and the economic impact of a pharmacist intervention were recorded.
\end{abstract}

Results: In this study, 178 out of 219 (81.2\%) patients received prophylactic treatments. Averagely, prophylactic therapy was compatible with standard treatment guidelines in $67.1 \%$ of cases. The implementation of ASHP guideline by a clinical pharmacist resulted in a cost saving of $>18,000$ USD monthly in this study, which would result in an estimated cost saving of $>216,000$ USD annually.

Conclusion: Although treatment guidelines are available for the prophylaxis of SRMD, failure to observe these guidelines could increase the cost of treatment and adverse effects. The clinical pharmacists' intervention in order to implement standard protocols has a significant impact on the reduction of unintended mistakes in prescribing prophylaxis, as well as significant cost savings. Keywords: acid suppression therapy, clinical pharmacy, histamine-2 receptor antagonist, proton pump inhibitor, stress ulcer prophylaxis

\section{Introduction}

Stress-related mucosal damage (SRMD) of gastrointestinal (GI) tract is a very common complication in critically ill patients, especially in cases with severe underlying disease. ${ }^{1}$ It is more common in the upper part of GI tract rather than the lower part. ${ }^{2}$ SRMD is described as the damage of gastric mucosa due to physiological stress and is a significant cause of mortality and morbidity in critically ill patients admitted to an intensive care unit (ICU). ${ }^{2}$

SRMD is usually detected in acid-producing areas of the stomach. Mechanical ventilation and coagulopathy are two major risk factors for this disease. While the
Correspondence: Ramin Niknam Gastroenterohepatology Research Center (GEHRC), P.O. Box 71935-131I, 9th floor, Research Tower, Namazi Hospital, Shiraz , Iran Tel/fax +98 7| 3628 I442

Email niknamramin@yahoo.com 
pathophysiology of the disease is multifactorial, it is mostly related to ischemia and hypoperfusion. In fact, following reduced blood circulation after ischemia, the balance between providing nutrients and mucosal defense and removing aggressive factors such as hydrochloric acid, infections, and free oxygen radicals would be impaired, which in turn leads to mucosal injury. ${ }^{3}$ Understanding the mechanism of SRMD has aided physicians and pharmacists to decide on appropriate therapies, which has led to a dramatic decrease in the incidence of the disease during recent years. ${ }^{4}$

Histamine-2-receptor antagonists and proton pump inhibitors are common regimens for stress ulcer prophylaxis (SUP). ${ }^{5}$ There are also some evidences on the effectiveness of beta adrenergic antagonists in preventing GI bleeding and decreasing its mortality rate. ${ }^{6}$

Many studies have revealed that a large percentage of patients admitted to non-ICU services do not need SUP medications while they receive them and also get discharged from hospital on these medications. ${ }^{7}$ The most recent guideline about prescribing SRMD prophylactic medications was published by the American Society of Health-System Pharmacists (ASHP) to be followed by physicians and pharmacists. $^{8}$

Clinical pharmacist is the one who can supervise prescribing such medications according to the available guidelines ${ }^{9}$ whose intervention has a remarkable effect on reducing drugrelated problems, improving clinical outcomes, decreasing length of hospital stay, and increasing economic benefits. ${ }^{10}$

The purpose of this study was to evaluate the clinical and economic impact of clinical pharmacists' interventions on the correction of SRMD prophylaxis practice. We also wished to identify and quantify the inappropriate use of SUP in these patients.

\section{Participants and Methods \\ Setting}

This was a prospective interventional study that was conducted over 6 months between September 2016 and March 2017 in Namazi and Shahid Faghihi hospitals. These teaching hospitals are under the supervision of Shiraz University of Medical Science, and they are the referral centers for the southern provinces of Iran, Fars. Daily medical rounds were performed by attending physicians, residents, general practitioners, medical interns, externs, and students every morning in these hospitals. After evaluating patients' conditions and considering paraclinical reports, required medical decisions were taken and new pharmacological prescriptions were ordered by attending physicians.
This study was conducted by following the guidelines outlined in the Declaration of Helsinki and Good Clinical Practice guideline. ${ }^{11,12}$ Institutional review board committee's approval was obtained from the Shiraz University of Medical Sciences Ethics Committee (92-01-05-6224). Patients provided written consents agreeing to be monitored over the period of assessment. This program was approved by the Pharmacy \& Therapeutics and Medical Executive Committee, which granted prescriptive authority to clinical pharmacists to initiate, modify, or discontinue SUP.

\section{Participants}

Patients who were admitted to Internal Medicine, General Surgery, and ICU wards of Namazi and Shahid Faghihi hospitals, which are major academic medical centers in Shiraz, Iran, were enrolled in this study. The following patients were excluded from the study: patients presented with peptic or duodenal ulcers during admission time, patients with incomplete medical files, patients who were discharged before 48 hours, and patients who were suffering from the Zollinger-Ellison syndrome or short bowel syndrome.

\section{Variables and measurements}

Required data that were collected in this study were comprised of demographic data (age and sex), patient's chief complaint, physician's diagnosis, length of hospital stay, admission date, discharge date, prescribed medications, drug administration route, drug interactions, drug regimen for SUP, patient past medical history, interventions performed for the patient, results of the interventions, laboratory and paraclinical results including international normalized ratio, platelet, serum creatinine, hematocrit, hemoglobin, white blood cell, liver function test, stool exam test, and the report of endoscopy procedure.

ASHP GI SUP guideline was used for evaluating the prescription of SUP medications. ${ }^{9}$ According to this guideline, risk factors are classified into two groups of major risk factors and minor risk factors, which take 1 and 0.5 scores, respectively. Patients with a total score of $\geq 1$ require to receive SUP medications, while patients with a total score of $<1$ do not require SUP (Table 1).

After detecting the risk factor score for each patient, the correlation of prescribed GI bleeding prophylactic medications with the obtained score was evaluated. Provided that the prescribed medication was correlated with the guideline, score 1 was considered for that patient, and in cases with no correlation between prescribed medications and guideline, 
Table I Gl bleeding prophylactic guideline for hospitalized patients

\begin{tabular}{|l|l|}
\hline $\begin{array}{l}\text { Severity of } \\
\text { risk factor }\end{array}$ & Risk factor \\
\hline Major & Coagulopathy (INR $>$ I.5 or PIt $\left.<50,000 / \mathrm{mm}^{3}\right)$ \\
\hline Major & Mechanical ventilation for $>48$ hours \\
\hline Major & $\begin{array}{l}\text { Patients with a history of GI ulceration or bleeding } \\
\text { within I year before admission }\end{array}$ \\
\hline Major & Glasgow coma score of $<10$ \\
\hline Major & Thermal injury of $>35 \%$ \\
\hline Major & Partial hepatectomy \\
\hline Major & Multiple trauma \\
\hline Major & Hepatic or renal transplantation \\
\hline Major & Spinal cord injury \\
\hline Major & Hepatic failure \\
\hline Minor & Sepsis \\
\hline Minor & ICU stay for $>$ I week \\
\hline Minor & Occult bleeding lasting at 6 days \\
\hline Minor & $\begin{array}{l}\text { High dose of corticosteroids }(250 \text { mg hydrocortisone } \\
\text { or equal) }\end{array}$ \\
\hline Minor & Using anti-PIt agents \\
\hline
\end{tabular}

Abbreviations: $\mathrm{Gl}$, gastrointestinal; ICU, intensive care unit; INR, internationa normalized ratio; Plt, platelet.

score 0 was selected. Moreover, in the cases that prophylactic medications were prescribed with no logical indication, intervention was made and ward resident was remarked about the pointlessness of prescribed SUP medications on the basis of the guideline.

\section{Statistical analysis}

Quantitative and qualitative data were described by mean $\pm \mathrm{SD}$ and frequency (percentage), respectively. The quantitative data were compared between the groups using chisquared test. All statistical analysis was done using SPSS version 22 . A $P$-value $<0.05$ was considered as statistically significant.

\section{Results}

A total number of 354 patients were enrolled in this study. Of them, 21 patients were excluded due to presenting active gastric or duodenal ulcer during hospital course, 92 cases were excluded due to being discharged from hospital before 48 hours of starting prophylactic medications, and 22 of them were excluded due to incomplete information.

Finally, 219 cases consisting of $117(53.4 \%)$ men and 102 $(46.6 \%)$ women were included in this study. Mean values of patients' laboratory data are shown in Table 2 . The mean age of the patients was $49.9 \pm 15.9$, and patients' ages ranged
Table 2 Mean value of patients' laboratory data

\begin{tabular}{|l|l|}
\hline Laboratory data & Mean \pm SD \\
\hline $\mathrm{Hb}(\mathrm{g} / \mathrm{dL})$ & $10.4 \pm 2.5$ \\
\hline $\mathrm{WBC}\left(10^{3} / \mu \mathrm{L}\right)$ & $8.4 \pm 3.5$ \\
\hline $\mathrm{S} . \mathrm{Cr}(\mathrm{mg} / \mathrm{dL})$ & $1.4 \pm 1.6$ \\
\hline $\mathrm{INR}$ & $1.5 \pm 0.8$ \\
\hline $\mathrm{PIt}\left(10^{3} / \mu \mathrm{L}\right)$ & $226.3 \pm 122.1$ \\
\hline
\end{tabular}

Abbreviations: $\mathrm{Hb}$, hemoglobin; INR, international normalized ratio; Plt, platelet; S.Cr, serum creatinine; WBC, white blood cell.

Table 3 Frequency and percentage of patients' underlying disease

\begin{tabular}{|l|l|l|}
\hline Underlying disease & Frequency $(\mathbf{n}=2$ I 9) & Percent \\
\hline Infectious & 25 & $\mathrm{II} .4$ \\
\hline Renal & 25 & $\mathrm{II} .4$ \\
\hline Respiratory & 20 & 9.1 \\
\hline Hepatogastrointestinal & 72 & 32.9 \\
\hline Trauma & 3 & 1.4 \\
\hline Cancer & 19 & 8.7 \\
\hline Post-organ transplantation & 13 & 5.9 \\
\hline Internal diseases & 42 & 19.2 \\
\hline
\end{tabular}

Table 4 Number and percentage of cases for whom prescribing gastrointestinal bleeding prophylactic medications was correlated with available guidelines

\begin{tabular}{|l|l|l|}
\hline Ward/number of patients & $\begin{array}{l}\text { Cases with } \\
\text { correlation } \\
\text { (number) }\end{array}$ & $\begin{array}{l}\text { Cases with } \\
\text { correlation } \\
\text { (percent) }\end{array}$ \\
\hline ICU/39 & 35 & 89.7 \\
\hline Internal medicine ward/I03 & 66 & 64.1 \\
\hline General surgery ward/77 & 46 & 59.7 \\
\hline Total/219 & 147 & 67.1 \\
\hline
\end{tabular}

Abbreviation: ICU, intensive care unit.

between 17 and 90 years. Of these 219 patients, 103 were admitted to the internal medicine ward, 77 were admitted to the general surgery ward, and 39 were admitted to the ICU.

In terms of frequency, patients' underlying diseases were hepatogastrointestinal disease, infectious disease, renal disease, respiratory disease, cancer, post-organ transplantation disease, and trauma (Table 3).

Correlation between prescribed GI bleeding prophylactic medications and available guidelines were $89.7 \%, 64.1 \%$, and $59.7 \%$ in ICU, internal medicine ward, and general surgery ward, respectively. There was a significant difference between correlation values in each ward ( $P$-value $=0.03$; Table 4$)$.

According to statistical analysis, there was a significant difference between correlation values of internal medicine ward and ICU $(P$-value $=0.005)$ and also between correlation 
Table 5 Evaluating the correlation between prescribed SRMD prophylactic medications and available guidelines

\begin{tabular}{|l|l|l|l|}
\hline Prophylaxis & $\begin{array}{l}\text { Cases } \\
\text { needed it }\end{array}$ & $\begin{array}{l}\text { Cases did not } \\
\text { need it }\end{array}$ & Total \\
\hline Cases received it & $116(52.9 \%)$ & $62(28.3 \%)$ & I78 (8I.2\%) \\
\hline $\begin{array}{l}\text { Cases did not } \\
\text { receive it }\end{array}$ & $10(4.5 \%)$ & $31(14.1 \%)$ & $4 I(I 8.8 \%)$ \\
\hline Total & $126(57.5 \%)$ & $93(42.5 \%)$ & $219(100 \%)$ \\
\hline $\begin{array}{l}\text { Total number of illogical } \\
\text { prescription of SRMD prophylactic } \\
\text { medications: 72 (32.9\%) }\end{array}$ & $\begin{array}{l}\text { Total number of logical } \\
\text { prescription of SRMD } \\
\text { prophylactic medications: I47 } \\
(67.1 \%)\end{array}$ \\
\hline
\end{tabular}

Abbreviation: SRMD, stress-related mucosal damage.

values of general surgery ward and ICU $(P$-value $=0.001)$. However, there was no significant difference between correlation values of internal medicine ward and general surgery ward $(P$-value $=0.65)$.

A total number of $126(57.5 \%)$ patients needed to receive prophylactic medications for SRMD according to the guidelines. In all, 25 (11.4\%) patients just had a minor risk factor, so there was no need to receive prophylactic medications. A total of 68 (31.1\%) patients had no risk factor and did not need to receive prophylactic medications either.

A total number of 116 (52.9\%) patients needed to receive prophylactic medications for SRMD according to the guidelines, and they were prescribed for them correctly. However, ten $(4.5 \%)$ patients needed to receive prophylactic medications for SRMD according to the guidelines too; they were not prescribed for them by the physicians. In all, 62 (28.3\%) patients did not need receiving prophylactic medications, but they were prescribed for them by their physicians. The remaining (14.1\%) patients were the cases who did not need receiving prophylactic medications, and they were not prescribed for them (Table 5).

Among 178 patients who received SRMD prophylactic medications, a total number of $135(75.8 \%)$ cases received pantoprazole, $40(22.5 \%)$ cases received ranitidine, and 3 $(1.7 \%)$ cases received omeprazole. There were no patients for whom two of these medications were prescribed. There was a significant difference between prescribing proton pomp inhibitors and histamine receptor antagonists $(P$-value $=0.02)$

Dosage and administration route of prescribed medications were determined according to the $\mathrm{ASHP}^{9}$ guideline and results are as follows. For 165 patients $(92.7 \%)$, dosage of prescribed medications was appropriate, while it was not the case for the other $31(7.3 \%)$ patients. A total number of $102(57.3 \%)$ patients received prophylactic medications on the basis of appropriate administration route in contrast to $76(42.7 \%)$ patients for whom it was inappropriate. In terms of SRMD prophylactic medication frequency, 95 (53.4\%) patients received it correctly, while 83 (46.6\%) patients did not. Totally, 87 (48.9\%) patients received SRMD prophylactic medications based on therapeutic protocols and guidelines, while 91 (51.1\%) patients did not.

According to the available guidelines, 97 (44.3\%) patients had one or more major risk factors for developing peptic ulcer and had to receive SRMD prophylactic medications. Moreover, 29 (13.2\%) patients had two or more minor risk factors for developing peptic ulcer and they had to receive SRMD prophylactic medications too. In all, 25 (11.4\%) patients had just a minor risk factor and $68(31.1 \%)$ patients had no risk factors for developing peptic ulcer, so they did not need to receive any SRMD prophylactic medications.

In this study, coagulopathy and mechanical ventilation $>48$ hours were considered as the most important risk factors for developing peptic ulcer.

After evaluating the prescribed SRMD prophylactic medications according to the guidelines, pharmacists remarked patients' physicians if the prescription had been illogical. Among 219 patients, 147 (67.1\%) patients did not need any interventions or remarking about prescribed medications. However, 72 (32.9\%) patients needed revision in their medications. Physicians accepted the pharmacist's recommendation for just 11 cases among these $72(15.2 \%)$ patients.

Total inpatient cost saving associated with discontinuation of all acid suppression therapies (proton pump inhibitors and histamine-2 receptor antagonists), which were administered inappropriately, was $\$ 18,000$ monthly, resulting in an estimated cost saving of $>\$ 216,000$ annually.

\section{Discussion}

SRMD prophylactic medications are widely prescribed all over the world. However, a lot of studies have revealed that a large percentage of patients admitted to non-ICU services do not need to receive these SRMD prophylactic medications. ${ }^{7}$ In the current study, a significant number of patients (28.3\%) received SRMD prophylactic medications while they did not need them according to the available guidelines for logical prescription of SRMD prophylaxis.

In a study that was conducted by Grube and May, the obtained results showed that $71 \%$ of patients received SRMD prophylactic medications with no logical indications. They concluded that prescribing SRMD prophylactic medications in general wards is not evidence based and should not be recommended. ${ }^{13}$ Their conclusion was the same as 
the results of Qadeer et $\mathrm{al}^{14}$ that showed that GI bleeding resulting from SRMD is not common in general wards, so prescribing SRMD prophylactic medications is not essential in such wards.

In a study conducted by Heidelbaugh and Inadomi, ${ }^{15}$ it was concluded that prescribing SRMD prophylactic medications should be according to available guidelines and illogical prescription of SRMD prophylactic medications causes additional cost and side effects for patients.

Prescription of any medications should be based on standard guidelines, especially ASHP guideline, ${ }^{9}$ which is the most used guideline about prescribing SRMD prophylactic medications. In the current study, $67.1 \%$ of patients received SRMD prophylactic medications according to the ASHP guideline, while $32.9 \%$ of patients did not. However, in a study conducted by Nardino et al, ${ }^{16} 54 \%$ of patients received SRMD prophylactic medications, while $64 \%$ of them did not need them according to the ASHP guideline.

Patients' different clinical situations, different methods of identifying discorrelation between prescribed medications and guidelines, and also attendance of clinical pharmacists in the ward may be the reasons of statistical differences among different studies.

It is estimated that the implementation of the ASHP guideline decreased the expenditure on drugs by $>18,000$ USD monthly in this study. According to a study that was conducted in 2015 in the same hospitals, the hospital expenditure rose due to the use of intravenous pantoprazole as one of the "Top-10" costly drugs. ${ }^{17}$

Choosing the best SRMD prophylactic medication is another important issue in this field. The ASHP guideline suggests to use anti-acids, histamine antagonists, surfactants, and proton pomp inhibitors as SRMD prophylactic agents. In the current study, physicians just prescribed histamine antagonists and proton pomp inhibitors; in that the prescription of proton pomp inhibitors was significantly higher than histamine antagonists $(P$-value $=0.02)$. In fact, 138 patients received proton pomp inhibitors, while 40 of them received histamine antagonists. Although there is not any documented evidence about the advantages of proton pomp inhibitors over histamine antagonists, currently proton pomp inhibitors are the most favorable medications for SRMD prophylaxis all over the world. ${ }^{18,19}$

The extensive prescription of proton pomp inhibitors, especially pantoprazole, may be due to the following reasons: drug resistance against histamine antagonists happens in the first 72 hours, lower rates of adverse events and drug interactions of pantoprazole, proton pomp inhibitors are the strongest anti-acid agents, and the minimum effect of proton pomp inhibitors is equal to that of histamine antagonists. ${ }^{20,21}$ However, as mentioned previously, there is no priority of any medications over the other one. In fact, the most suitable medication is the one that is more cost-effective in that specific situation. ${ }^{22}$

In this study, clinical pharmacists recommended logical prescription of medications in cases where prescribing SRMD prophylactic medications did not match with the ASHP guideline. Among 72 patients, who needed clinical pharmacy intervention, only in $11(15.2 \%)$ cases, the physicians accepted clinical pharmacists' recommendation. The effect of clinical pharmacy interventions and the acceptance rate of their recommendations are different among different studies. There are numerous evidences on that clinical pharmacy interventions would reduce healthcare costs and also improve quality of healthcare services..$^{9,10,17}$

This study had some limitations. First, it was conducted at two centers with a limited number of participants. Second, it was performed over a period of 6 months, so our patients may not have been completely representative of any hospital settings. Third, in this study, we did not have a control group. Longer term cost-effective analyses was outside the scope of this study.

\section{Conclusion}

Considering all the mentioned results, it is concluded that well-trained clinical pharmacists can be involved in patients' care to help treat patients more cost-effectively, which leads to a reduction in healthcare costs and improves quality of healthcare services. It seems necessary to establish and use SRMD prophylaxis guideline and registry-based information of SRMD prophylaxis, particularly in an ICU setting, to improve the quality of care for these patients.

In fact, clinical pharmacists are complementary members in each treatment team and employing them would have so many benefits for both patients and healthcare systems.

\section{Acknowledgment}

This work was a part of a Pharm.D thesis written by Rahim Mohammadi and was supported by the Research Council of Shiraz University of Medical Sciences, Shiraz, Iran (No. 92-01-05-6224).

\section{Disclosure}

The authors report no conflicts of interest in this work.

\section{References}

1. Peura DA. Stress-related mucosal damage: an overview. Am J Med. 1987;83(6A):3-7. 
2. Peivandi Yazdi A, Imantalab M. Stress-related mucosal disease in critically ill patients. Patient Saf Qual Improv. 2015;3(2):266-268.

3. Sesler JM. Stress-related mucosal disease in the intensive care unit: an update on prophylaxis. AACN Adv Crit Care. 2007;18(2):119-128.

4. Spirt MJ. Stress-related mucosal disease: risk factors and prophylactic therapy. Clin Ther. 2004;26(2):197-213.

5. Miller TA. Mechanisms of stress-related mucosal damage. Am J Med. 1987;83(6A):8-14.

6. Poynard T, Calès P, Pasta L, et al. Beta-adrenergic-antagonist drugs in the prevention of gastrointestinal bleeding in patients with cirrhosis and esophageal varices. An analysis of data and prognostic factors in 589 patients from four randomized clinical trials. Franco-Italian Multicenter Study Group. N Engl J Med. 1991;324(22):1532-1538.

7. Heidelbaugh JJ, Inadomi JM. Magnitude and economic impact of inappropriate use of stress ulcer prophylaxis in non-ICU hospitalized patients. Am J Gastroenterol. 2006;101(10):2200-2205.

8. ASHP therapeutic guidelines on stress ulcer prophylaxis. ASHP Commission on therapeutics and approved by the ASHP Board of Directors on November 14, 1998. Am J Health Syst Pharm. 1999;56(4):347-379.

9. Hanlon JT, Weinberger M, Samsa GP, et al. A randomized, controlled trial of a clinical Pharmacist intervention to improve inappropriate prescribing in elderly outpatients with polypharmacy. Am J Med. 1996;100(4):428-437.

10. Viktil KK, Blix HS. The impact of clinical pharmacists on drug-related problems and clinical outcomes. Basic Clin Pharmacol Toxicol. 2008;102(3):275-280.

11. World Medical Association Declaration of Helsinki Ethical Principles for Medical Research Involving Human Subjects. Bulletin of the World Health Organization, 2001, 79(4).

12. ICH harmonized tripartite guideline: Guideline for Good Clinical Practice. J Postgrad Med. 2001 Jan-Mar;47(1):45-50.
13. Grube RR, May DB. Stress ulcer prophylaxis in hospitalized patients not in intensive care units. Am J Health Syst Pharm. 2007;64(13): 1396-1400.

14. Qadeer MA, Richter JE, Brotman DJ. Hospital-acquired gastrointestinal bleeding outside the critical care unit: risk factors, role of acid suppression, and endoscopy findings. J Hosp Med. 2006;1(1):13-20.

15. Heidelbaugh JJ, Inadomi JM. Magnitude and economic impact of inappropriate use of stress ulcer prophylaxis in non-ICU hospitalized patients. Am J Gastroenterol. 2006;101(10):2200-2205.

16. Nardino RJ, Vender RJ, Herbert PN. Overuse of acid-suppressive therapy in hospitalized patients. Am J Gastroenterol. 2000;95(11): 3118-3122.

17. Mahmoudi L, Karamikhah R, Mahdavinia A, Samiei H, Petramfar P, Niknam R. Implementation of pharmaceutical practice guidelines by a project model based: clinical and economic impact. Medicine (Baltimore). 2015;94(42):e1744.

18. Sung JJ. The role of acid suppression in the management and prevention of gastrointestinal hemorrhage associated with gastroduodenal ulcers. Gastroenterol Clin North Am. 2003;32(3 Suppl):S11-S23.

19. Heidelbaugh JJ, Goldberg KL, Inadomi JM. Overutilization of proton pump inhibitors: a review of cost-effectiveness and risk [corrected]. Am J Gastroenterol. 2009;104 (Suppl 2):S27-S32.

20. Beejay U, Wolfe MM. Acute gastrointestinal bleeding in the intensive care unit. The gastroenterologist's perspective. Gastroenterol Clin North Am. 2000;29(2):309-336.

21. Netzer P, Gaia C, Sandoz M, et al. Effect of repeated injection and continuous infusion of omeprazole and ranitidine on intragastric $\mathrm{pH}$ over 72 hours. Am J Gastroenterol. 1999;94(2):351-357.

22. Allen ME, Kopp BJ, Erstad BL. Stress ulcer prophylaxis in the postoperative period. Am J Health Syst Pharm. 2004;61(6):588-596.
ClinicoEconomics and Outcomes Research

\section{Publish your work in this journal}

ClinicoEconomics and Outcomes Research is an international, peerreviewed open-access journal focusing on health technology assessment, pharmacoeconomics and outcomes research in the areas of diagnosis, medical devices, and clinical, surgical and pharmacological intervention. The economic impact of health policy and health systems

\section{Dovepress}

organization also constitute important areas of coverage. The manuscript management system is completely online and includes a very quick and fair peer-review system, which is all easy to use. Visit http://www.dovepress.com/testimonials.php to read real quotes from published authors. 